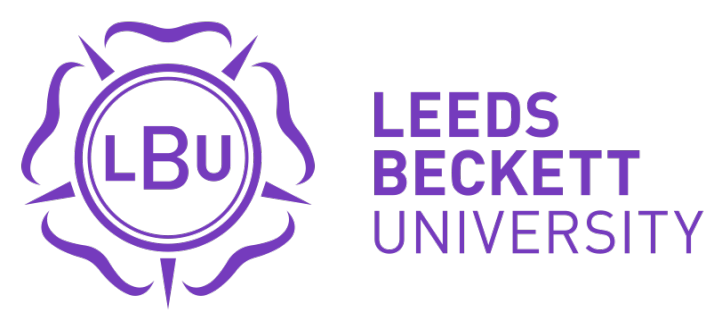

Citation:

Beggs, CB and Shepherd, S and Zamboni, P (2014) Cerebral venous outflow resistance and interpretation of cervical plethysmography data with respect to the diagnosis of chronic cerebrospinal venous insufficiency. Phlebology / Venous Forum of the Royal Society of Medicine, 29 (3). 191 - 199. ISSN 0268-3555 DOI: https://doi.org/10.1258/phleb.2012.012039

Link to Leeds Beckett Repository record:

https://eprints.leedsbeckett.ac.uk/id/eprint/2375/

Document Version:

Article (Accepted Version)

The aim of the Leeds Beckett Repository is to provide open access to our research, as required by funder policies and permitted by publishers and copyright law.

The Leeds Beckett repository holds a wide range of publications, each of which has been checked for copyright and the relevant embargo period has been applied by the Research Services team.

We operate on a standard take-down policy. If you are the author or publisher of an output and you would like it removed from the repository, please contact us and we will investigate on a case-by-case basis.

Each thesis in the repository has been cleared where necessary by the author for third party copyright. If you would like a thesis to be removed from the repository or believe there is an issue with copyright, please contact us on openaccess@leedsbeckett.ac.uk and we will investigate on a case-by-case basis. 


\section{Cerebral venous outflow resistance and interpretation of cervical plethysmography data with respect to the diagnosis of chronic cerebrospinal venous insufficiency.}

Clive Beggs ${ }^{1}$, Simon Shepherd ${ }^{1}$ and Paolo Zamboni ${ }^{2}$

${ }^{1}$ Medical Biophysics Laboratory, University of Bradford, UK.

${ }^{2}$ Vascular Diseases Centre, University of Ferrara, Italy.

\section{Corresponding Author: $\quad$ Clive B. Beggs, PhD}

Prof Clive Beggs

Medical Biophysics Laboratory

School of Engineering, Design \& Technology

University of Bradford

Bradford

West Yorkshire

BD7 1DP

United Kingdom

email: c.b.beggs@bradford.ac.uk, Tel: +44(0)1274 233679, Fax: +44(0)1274 234124

Short title: Cerebral venous outflow and cervical plethysmography

Key words: plethysmography; cerebral venous system; venous haemodynamics; chronic cerebrospinal venous insufficiency; multiple sclerosis

\section{Disclosures}

Clive Beggs and Simon J Sheperd have nothing to disclose.

Paolo Zamboni received technical equipments from MicroLab Padua, Italy, and Esaote Biomedica Genoa Italy.

The Vascular Disease Center-University of Ferrara received for this study technical equipment from ESAOTE Biosound, Genoa-Italy. 


\section{ABSTRACT}

Objective: While chronic cerebrospinal venous insufficiency (CCSVI) can be characterised using cervical plethysmography, much remains unknown about the haemodynamics associated with this procedure. The study aim was therefore to gain a deeper understanding of the observed haemodynamics.

Method: 40 healthy controls and 44 CCSVI patients underwent cervical plethysmography, which involved placing a strain-gauge collar around their necks and tipping them from the upright $\left(90^{\circ}\right)$ to supine position $\left(0^{\circ}\right)$ in a chair. Once stabilized, they were returned to the upright position, allowing blood to drain from the neck. A mathematical model was used to calculate the hydraulic resistance of the extracranial venous system for each subject in the study.

Results: The mean hydraulic resistance of the extracranial venous system was 10.28 (SD 5.14) $\mathrm{mmHg} . \mathrm{s} / \mathrm{mL}$ in the healthy controls and 16.81 (SD 9.22) in the CCSVI patients $(p<0.001)$.

Conclusions: The haemodynamics of the extracranial venous system are greatly altered in CCSVI patients.

(150 words)

\section{Introduction}

In a recent study, using strain-gauge cervical plethysmography (SGP) we demonstrated that chronic cerebrospinal venous insufficiency (CCSVI) is associated with altered cerebral venous drainage dynamics ${ }^{1}$. SGP is a noninvasive technique that measures variations in electrical resistance associated with changes in blood volume, recorded by the means of a sensor encircling any cylindrical segment of the body ${ }^{2,3}$. Although widely used to detect venous abnormalities in the legs by measuring change in venous volume in relation to change in posture and exercise ${ }^{2}$, it has never before been used in the context of CCSVI. We decided to use SGP instead of photoplethysmography (PPG) because individuals with CCSVI exhibit great variability in the venous drainage pathways from the brain. SGP allowed us to acquire a total measurement of the whole circumference of the neck, including the main and collateral venous routes, whereas PPG would have involved the use of probes of a limited size and therefore could only be used to explore part of the neck, or just one side. In addition, the fastening system associated with PPG was not as well suited to the tilt test as the SGP collar. In our study the vast majority of the patients had CCSVI, which was associated with multiple sclerosis (MS) (95\%). While others have identified morphological abnormalities in the cerebral venous drainage pathways of MS patients, using echo colour Doppler sonography (ECDS) ${ }^{4-8}$ and magnetic resonance imaging (MRI) ${ }^{9-12}$, it has not been possible to characterize the dynamics of the overall drainage system using these techniques. For example, although the physical dimensions of individual veins can be determined using ECDS, together with abnormalities such as stenotic lesions, it is only possible, using this technology, to examine isolated sections of the venous drainage system. ECDS cannot be used to quantify the dynamics of the entire cerebral drainage system. Furthermore, in a recent meta-analysis of screening studies for CCSVI, Laupacis et al ${ }^{13}$ found considerable heterogeneity in results obtained using ECDS. The reasons for this heterogeneity include factors such as; absence of studies that describe what is normal in the cerebral venous return; absence of any consensus regarding which parameters should be measured; and 
lack of consensus regarding the correct protocol to use. While a recently published consensus document ${ }^{14}$ should help to improve things with regard to the ECDS protocol, this cannot overcome operator dependent factors which might influence the results of a diagnostic test, such as the pressure of the probe on the neck, or assist with the interpretation of any collateral circulation and flow anomalies that might be found. However, through the use of SGP it is possible to quantify and characterize the overall venous drainage rate, irrespective of the physical location of any stenotic lesions. Indeed, through SGP we have been able to demonstrate that the morphological abnormalities associated with CCSVI, such as stenosis of the internal jugular veins (IJVs) and azygos vein, have a profound effect on the overall dynamics of the cerebral venous drainage system.

CCSVI is generally diagnosed using ECDS of the extracranial veins. However, due to the floppiness of the vessels involved and the variability of the venous vasculature, this technique can be highly subjective and open to misinterpretation ${ }^{15}$. Indeed, variability arising from venous sonography has caused some researchers to doubt the existence of CCSVI 16, 17. However, through the use of strain-gauge cervical plethysmography we have been able to demonstrate that CCSVI appears to be a real clinical phenomenon ${ }^{1}$ and also show that in patients with MS the condition can be diagnosed with reasonable accuracy (selectivity $=84 \% ; p<0.0001)$. Being a relatively simple procedure, which avoids much of the subjectivity associated with venous sonography, SGP appears to have potential as an inexpensive non-invasive technique for diagnosing CCSVI.

While the results of our initial study were promising, much remains unknown about the use of cervical plethysmography for diagnosing CCSVI, and there is need for additional work to understand how the technique may be best utilized. In particular, relatively little is known about the interpretation of plethysmography plots, and of how these relate to the dynamics of the cerebral venous drainage system. In this paper we examine this issue and investigate the physical science that underpins cervical plethysmography, with the aim of better understanding the haemodynamics associated with the technique.

\section{Method}

We assessed by the means of cervical plethysmography 44 CCSVI patients (95\% MS and 5\% healthy controls) and 40 subjects negative to ECDS screening for CCSVI (95\% healthy controls and $5 \%$ with MS). The group of CCSVI negative subjects (hereafter referred to as healthy controls) was matched for age with CCSVI positive (mean age 41.5 , std $=14.4$ years vs. mean age of 41.0 , std $=12.1$ years, respectively, $\mathrm{p}=\mathrm{NS}$ ) and also for gender (18 females and 22 males vs 25 females and 19 males, respectively, $\mathrm{p}=\mathrm{NS}$ ). The entire cohort was informed about the methods and purpose of the experimental procedure and agreed to participate by signing an informed consent form. This prospective study was in accordance with Ethical Standards of the Committee on Human Experimentation of the University of Ferrara.

In order to gain new insights into the dynamics of the cervical plethysmography process we created a simple mathematical model of the extracranial venous system with the aim of interpreting the plethysmography plots. We applied this model to plethysmographic data acquired by Zamboni et al ${ }^{1}$ (see Table 1 ) in order to calculate the overall hydraulic resistance of the extracranial venous system. In Zamboni et al's study, participants were placed in a tilt chair (Figure 1) in the upright position and a passive cervical collar was placed around their necks. This collar comprised a thin silicone rubber tube containing electrically conductive 
indium-gallium liquid metal, which acted as the sensor (Strain-gauge plethysmometer, Microlab, Padua, Italy). The silicone used is particularly soft and special attention was given to the choice of collar size so that it adhered to the skin surface without creating unwanted compression of the underlying vessels. The neck circumference of each subject was measured individually before each test and a collar chosen (ranged from 25 to $35 \mathrm{~cm}$ ) that best fit the circumference of the neck. The collar utilized a special silicone rubber, which ensured good adhesion to the skin, thus avoiding the need for any string adjustment. A platinum electrode was inserted at each end of the tube to form an electrical circuit, which was connected to a sensitive data logger. As the collar stretched, it both elongated and reduced in cross-sectional area, thus increasing the electrical resistance of the circuit. By monitoring changes in electrical resistance of the circuit, it was possible to indirectly measure the cervical venous blood volume ${ }^{18}$.

After the collar was fitted, the study subjects were instructed not to move their neck or to swallow during the procedure. An angular position sensor fitted to the chair was used to record the chair angle from the upright position throughout the procedure (i.e. dotted line in Figure 2). After calibration, the subjects were tipped from the upright position $\left(90^{\circ}\right)$ into the supine position $\left(0^{\circ}\right)$, which caused the blood in the jugular veins to greatly increase in volume (i.e. solid line in Figure 2). Once the blood volume stabilized, the chair was tilted forward again into the upright position $(<1.8 \mathrm{sec})$, allowing the blood to rapidly drain from the neck. For each subject, this procedure was repeated three times and the average of the three sets of data recorded. An interval of $3 \mathrm{~min}$ was enforced between the procedures to allow for blood redistribution. For each experimental run the following key parameters were recorded: the venous volume (VV); the filling time (FT) required to achieve $90 \%$ of $\mathrm{VV}(90 \% \mathrm{VV})$; the residual volume (RV); and the emptying time (ET) required to achieve $90 \%$ of EV $(90 \% E V)$, where EV $=\mathrm{VV}-\mathrm{RT}$.

For each participant in the study, the mean values of the key parameters outlined above were used to determine two metrics: (i) the gradient of the filling curve, FG; and (ii) the gradient of the emptying curve, EG, as follows:

$$
\begin{aligned}
& F G=\frac{(0.9 \times V V)}{F T} \\
& E G=\frac{[0.9 \times(V V-R V)]}{E T}
\end{aligned}
$$

For ease of reference a summary of the results of Zamboni et al's study are presented in Table 1. Readers who seek more details about the methodology used are directed to Zamboni et al ${ }^{1}$. 
Table I. Results of plethysmography study ${ }^{1}$

\begin{tabular}{|c|c|c|c|c|c|c|c|c|}
\hline & & $\begin{array}{l}\text { Controls } \\
(n=40)\end{array}$ & $\begin{array}{l}\text { Controls } \\
(n=40)\end{array}$ & $\begin{array}{l}\text { Controls } \\
(n=40)\end{array}$ & $\begin{array}{l}\text { CCSVI } \\
(n=44)\end{array}$ & $\begin{array}{l}\text { CCSVI } \\
(n=44)\end{array}$ & $\begin{array}{l}\text { CCSVI } \\
(n=44)\end{array}$ & \\
\hline $\begin{array}{c}\text { Variable } \\
\text { VV }\end{array}$ & $\begin{array}{c}\text { Units } \\
\mathrm{mL}\end{array}$ & $\begin{array}{c}\text { Mean } \\
5.32\end{array}$ & $\begin{array}{c}\text { St. Dev. } \\
2.37\end{array}$ & $\begin{array}{l}\text { Range } \\
1.67- \\
10.10\end{array}$ & $\begin{array}{l}\text { Mean } \\
5.66\end{array}$ & $\begin{array}{c}\text { St. Dev. } \\
2.36\end{array}$ & $\begin{array}{c}\text { Range } \\
2.00- \\
12.30\end{array}$ & $\begin{array}{c}\text { p value } \\
0.520\end{array}$ \\
\hline FT & Seconds & 5.81 & 1.99 & $\begin{array}{l}1.50- \\
11.10\end{array}$ & 4.45 & 2.16 & $\begin{array}{l}1.07- \\
10.54\end{array}$ & 0.003 \\
\hline FG & $\mathrm{mL} / \mathrm{sec}$ & 0.92 & 0.45 & $\begin{array}{c}0.28- \\
2.25\end{array}$ & 1.50 & 0.85 & $\begin{array}{c}0.43- \\
3.76\end{array}$ & $<0.001$ \\
\hline RV & $\mathrm{mL}$ & 0.54 & 1.31 & $\begin{array}{c}-1.93- \\
4.47\end{array}$ & 1.37 & 1.34 & $\begin{array}{c}-1.27- \\
4.60\end{array}$ & 0.005 \\
\hline EV & $\mathrm{mL}$ & 4.78 & 1.85 & $\begin{array}{l}2.17- \\
10.00\end{array}$ & 4.28 & 1.99 & $\begin{array}{l}1.60- \\
10.27\end{array}$ & 0.241 \\
\hline ET & Seconds & 1.84 & 0.54 & $\begin{array}{c}0.41- \\
2.98\end{array}$ & 2.66 & 0.95 & $\begin{array}{c}1.13- \\
5.21\end{array}$ & $<0.001$ \\
\hline EG & $\mathrm{mL} / \mathrm{sec}$ & 2.73 & 1.63 & $\begin{array}{c}0.85- \\
8.94\end{array}$ & 1.73 & 0.94 & $\begin{array}{c}0.50- \\
4.27\end{array}$ & 0.001 \\
\hline Age & Years & 41.45 & 14.40 & $\begin{array}{c}20.00- \\
72.00\end{array}$ & 41.02 & 12.12 & $\begin{array}{c}17.00- \\
67.00\end{array}$ & 0.883 \\
\hline
\end{tabular}

\section{Model}

In the model it is assumed that when the study subjects are tipped into the supine position, an unknown volume of blood collects in the cervical veins above the silicone collar. When returned to the upright position, due to the action of gravity, this bolus of blood drains away, at a rate, $Q \mathrm{~mL} / \mathrm{s}$, which is equal to the emptying gradient, EG, through the extracranial venous system. In the model the relationship between flow rate, pressure and hydraulic resistance is as follows:

$$
Q=\frac{\Delta P}{R}
$$

Where; $Q$ represents the blood flow rate from the bolus of trapped blood $(\mathrm{mL} / \mathrm{s}) ; \Delta P$ represents the pressure drop (gradient) across the whole extracranial venous system $(\mathrm{mmHg})$; and $R$ represents the hydraulic resistance of the extracranial venous system $(\mathrm{mmHg} . \mathrm{s} / \mathrm{mL})$. When upright, the pressure drop across the venous system can be determined as follows:

$$
\Delta P=\rho g h
$$

Where; $\rho$ is the density of blood (i.e. $1060 \mathrm{~kg} / \mathrm{m}^{3}$ ); $h$ is the average height of the column of blood from the point where the IJV leaves the cranium to the heart (i.e. $280 \mathrm{~mm}$ ); and $g$ is the acceleration due to gravity (i.e. $9.81 \mathrm{~m} / \mathrm{s}^{2}$ ).

Because the cerebral/extracranial venous system is one continuous sealed system, in the model we assumed that as the bolus of trapped venous blood discharges, the pressure head, $\Delta P$, remains constant while its volume changes (see Figure 3). Therefore by substituting equation 4 into equation 3 it is possible to calculate the hydraulic resistance, $R$, of the extracranial venous system during the plethysmography discharge procedure. In order to calculate the hydraulic resistance we assumed that when the subjects are 
returned in the upright position, the height of the column of extracranial venous blood, $h$, is on average 280 $\mathrm{mm}$, for all the individuals in the study.

\section{Results}

From the experimental results presented in Table 1 it can be seen that there is a strong significant difference ( $p=0.001$ ) between the CCSVI patients and the healthy controls for the metrics $F G$ and $E G$. By comparison no significant difference exists for the parameters VV and EV, suggesting great variability within both study cohorts. This is illustrated by the sample time-series plethysmography plots for CCSVI patients and healthy controls presented in Figure 4. From these it can be seen that while the general shape of all the graphs is similar, there is great variation between the plots produced by the individual subjects, making discrimination between the two cohorts difficult. The most consistent distinguishing feature is the emptying gradient, EG, which is almost vertical in the controls and is shallower in the group of MS patients. The other distinguishing feature is the value of the residual volume, RV. This is generally much lower in the controls compared with the MS patients, although some MS patients (e.g. Patient 3) also exhibited a low RV value. While the results in Table 1 clearly show a strong statistical difference between the cohorts for $F G$, this is not obvious from the samples shown in Figure 4. Having said this, the difference may be explained by the oscillatory part of the graph, which appears to have a greater slope in the controls compared with the CCSVI patients, something that would explain the longer FT required to achieve $90 \%$ of VV in the controls.

Table 2 shows the results of the analysis using equations 3 and 4 above. From this it can be seen that the hydraulic resistance is on average $63.5 \%$ greater in the MS patients compared with the controls $(p<0.001)-$ something which explains why the venous blood drains away much more slowly in the CCSVI patients compared with the controls.

Table 2. Results of analysis - mean values and (standard deviation)

$\begin{array}{cccc}\text { Cohort } & \text { No. } & \begin{array}{c}\text { EG } \\ \text { (mL/sec) }\end{array} & \begin{array}{c}\text { Calculated } \\ \text { Resistance } \\ \text { (mmHg.s/mL) }\end{array} \\ \text { Controls } & 40 & 2.73(1.63) & 10.28(5.14) \\ \text { CCSVI patients } & 44 & 1.73(0.94) & 16.81(9.22) \\ \text { p value } & - & 0.001 & <0.001\end{array}$

\section{Discussion}

The venous system is a complex, low pressure, free communicating network of vessels, which contains $75 \%$ of the body's circulating blood. When subjects lie in the supine position, the absence of any gravitational gradient causes the blood to be more evenly distributed throughout the system, with the result that the venous blood tends to collect in the jugular veins causing their volume to greatly increase. However when upright, the distribution changes and approximately $70 \%$ of the blood volume becomes located below the diaphragmatic line, due to the action of gravity ${ }^{19}$. Consequently the jugular veins, being particularly floppy vessels, tend to collapse when upright. Through the use of a passive strain-gauge collar we have been able to accurately measure this change in volume and to produce plethysmography plots such as those in figures 
2 and 4. From these it can be seen that the filling process comprises two stages: an initial, steep, approximately linear phase, which is followed by an oscillatory phase, which has a shallow gradient. The initial phase corresponds with the period when the collapsed veins fill with blood until the vessels eventually become full. Once the vessels are full, the oscillations then commence. These appear to be periodic in nature, oscillating at approximately $1 \mathrm{~Hz}$ (see Figure 5), roughly cardiac frequency, suggesting that the distention of the IJVs during this period might be due to the pulsatility of the venous blood flow from the brain. Evidence supporting this opinion comes from Ambarki et al ${ }^{20}$ and Raksin et al ${ }^{21}$, who observed, using MRI of the cervical veins, a venous pulse similar in shape and periodicity to that exhibited by the oscillations in figures 4 and 5. However, given the irregular nature of the oscillations in some individuals (e.g. Control 1 in Figure 4(a)), it would appear that other unknown mechanisms might also be at work.

When an open vessel drains, the discharge flow rate decays exponentially with time as the height of the column of fluid decreases. However during the plethysmography process, because the venous system is a closed system, the height of the column of blood does not change, rather the sides of the distended blood vessels contract as the trapped blood drains away (see Figure 3). Consequently, our model predicts that the discharge blood flow rate should be linear. From Figure 4 it can be seen that, for both cohorts, this is indeed the case, with the plethysmography plots showing a straight line for much of the discharge process, as predicted by the model. It can also be seen that the linear discharge rate (i.e. EG) is much greater in the healthy controls $(2.73 \mathrm{~mL} / \mathrm{s})$ compared with the CCSVI patients $(1.73 \mathrm{~mL} / \mathrm{s})$ - a result that is strongly significant $(p=0.001)$. This strongly implies the presence of impaired cerebral venous drainage in the cohort of CCSVI patients - something quantified by the calculated hydraulic resistance in the upright position, which is on average $63.5 \%$ greater in the CCSVI patients. As such, our work appears to corroborate that of Monti et al ${ }^{22}$, who found reduced cerebral venous outflow in the upright position to be strongly associated $(p<0.0001)$ with MS. Given that CCSVI has been shown by many researchers to be associated with MS $4-8$, 23, 24, this suggests the presence of abnormal cerebral venous drainage hydrodynamics in many patients with MS ${ }^{22}$.

By nature fluids take the path of least resistance. If main pathways, such as the IJVs, become occluded, then the upstream pressure will increase until it is great enough to force the venous blood through alternative collateral routes. While these collaterals allow the venous blood to drain away, they are more torturous than the original main pathways and so the hydraulic resistance of the whole system tends to increase. This is illustrated in Figure 6, which shows collateral flow through the thyroid veins in a MS patient due to $95 \%$ stenosis of the left IJV. Studies by Zamboni et al ${ }^{25}$ have shown that CCSVI patients exhibit a wide degree of variance with respect to collateral rerouting. Consequently, one would expect the hydraulic resistance in these patients to exhibit greater variability than in healthy controls - something observed in the results in Table 2. Indeed, the high degree of variability exhibited by individuals with CCSVI and the torturous nature of the collateral pathways, make it very difficult to accurately calculate hydraulic resistance. This is because conventional hydraulic analysis techniques tend to struggle with systems that have numerous branches and vessels that are distended and distorted.

Because of collateral rerouting, one would expect the mean transit time of the blood through the extracranial venous system to increase in the CCSVI patients compared with healthy controls. From equation 3 it can be seen that as the hydraulic resistance of the system increases, so the venous blood flow rate will decrease. This in turn will tend to reduce the velocity of the blood flow, increasing the transit time. Furthermore, because the collateral pathways are generally longer than the original routes, this will also tend to increase 
the transit time of the venous blood flow. In a study involving 103 MS patients and 42 healthy controls, Mancini et al 24 found the mean tissue-vein transit time to be $3.2 \mathrm{sec}$ in the MS cohort, compared with only $2.9 \mathrm{sec}$ in the controls $(p=0.037)$. Given that $95 \%$ of the CCSVI patients in our study also had MS, our findings would appear to support those of Mancini et al. Interestingly, although Mancini et al found CCSVI to be present in $77 \%$ of the MS patients studied, they found no difference in the cerebral circulation time between the CCSVI positive and CCSVI negative MS patients. While this finding is difficult to explain, it does suggest that vascular changes other than CCSVI may be influencing the transit of cerebral blood in MS patients. Evidence supporting this hypothesis comes from Zivadinov et al 23 who observed a marked reduction in cerebral veins $<0.3 \mathrm{~mm}$ diameter in MS patients compared with controls, which statistically correlated with a diagnosis of CCSVI ( $p<0.003$ ). Law et al ${ }^{26}$, Ge et al ${ }^{27}$ and Varga et al ${ }^{28}$ also observed reductions in cerebral blood flow in the white matter of MS patients, strongly suggesting alterations in the cerebral vascular bed in patients with MS.

While the linear model above appears to explain much of the dynamics of the venous discharge, it is noticeable that for many of the individuals in the study (e.g. CCSVI patients 1 and 2) the linear discharge phase is followed a secondary phase that is not predicted by the model. In these individuals the initial linear discharge period is followed by a second oscillatory period in which the drainage rate is much slower. This discharge pattern, which appears to be particularly prevalent amongst the CCSVI patients (as indicated by the high mean residual volume (RV) value for this cohort), is difficult to explain. However, the oscillatory nature of the signal implies the presence of a significant volume of blood in the cervical veins, suggesting that they have not emptied. This implies that the flow rate attempting to pass through the venous drainage system in these individuals exceeds its discharge capacity. Consequently, after an initial period of rapid discharge, the system becomes 'swamped' with the result that the blood backs up in the jugular veins and is only able to drain away slowly. For example, this pattern of drainage can clearly be observed in CCSVI Patient 1 in Figure 4(b).

One unexpected finding of the study was that the FG was markedly steeper in the CCSVI patients compared with the healthy controls, primarily due to reduced FT. While at first sight this phenomenon might appear more difficult to explain than the shallower EG, it too is consistent with impaired flow in the extracranial venous pathways. In the supine position, the rate at which blood accumulates above the collar (i.e. FG) is dependent both on the rate at which arterial blood enters the cranium and the rate at which it drains away through the veins back to the heart. Therefore, if for any reason, flow through the extracranial venous pathways is impaired, as would be the case in CCSVI patients, then the blood would tend to accumulate in the neck at a faster rate, resulting in a reduced FT and a steeper FG.

One common mistake often made by those unfamiliar with the discipline of fluid mechanics, is to confuse the morphological features of the vascular system with its hydrodynamic behaviour. Although the two are closely related, they are separate and distinct entities. While one might be able to identify the presence of stenotic lesions or incompetent valves at specific locations in the extracranial venous system using ECDS, it is not possible from this alone to calculate the hydraulic resistance of the whole system. This is because the hydraulic resistance of the extracranial venous system is influenced by many factors, including; blood velocity; the presence of turbulence; the length and diameter of collateral pathways; and the smoothness and uniformity of the vessels. Two venous anomalies in separate patients which appear morphological similar with ECDS, might in reality impact on the blood flow dynamics in very different ways, depending on other factors. Failure to appreciate this can result in erroneous conclusions about the behaviour of the 
venous system. Given this, strain-gauge cervical plethysmography appears to have great potential. Using this new methodology, it is possible for the first time to calculate the overall hydraulic resistance of the extracranial venous system, and thus obtain a quantifiable metric that can be used to objectively compare blood flow both in CCSVI patients and in health individuals. As such, the method is a useful addition to ECDS - especially when it is considered that venous sonography is a highly specialized discipline, that if applied incorrectly can yield erroneous results ${ }^{29}$.

\section{Conclusion}

Although many studies have shown CCSVI to be associated with morphological abnormalities of the extracranial venous pathways ${ }^{4-8,25}$, because of the limitations of ECDS it has not been possible to assess how these might influence the overall dynamics of the extracranial venous system. Because the hydraulic resistance attributable to any given stenosis cannot be determined using ECDS, it is impossible using this sonography to assess the overall haemodynamic behaviour of the extracranial venous system. This is particularly the case, when one considers that CCSVI frequently involves the collateral rerouting of the venous blood back to the heart ${ }^{25}$. Consequently, interventionalists who might perform venous angioplasty on perceived blockages cannot be sure how this intervention will affect the dynamics of the whole system. Through the use of cervical plethysmography, it should be possible to quantifiably assess this. Using the methodology described above, it is possible to calculate the hydraulic resistance of the extracranial venous system for each individual tested. This is a significant step forward because it allows, for the first time, noninvasive quantifiable assessment of the hydrodynamics associated with CCSVI to be made, in addition to any examination of morphological abnormalities using ECDS. Given that the process appears to be a readily repeatable procedure, much less prone to operator error compared with echo colour Doppler ultrasound, we believe that cervical plethysmography has great potential as an inexpensive tool for diagnosing venous drainage abnormalities and urge that further research be carried in this much-neglected field.

\section{References}

1. Zamboni $\mathrm{P}$, Menegatti $\mathrm{E}$, Conforti $\mathrm{P}$, et al. Assessment of cerebral venous return by a novel plethysmography method. Journa of Vascual Surgery:(in press).

2. Nicolaides AN. Investigation of chronic venous insufficiency: A consensus statement (France, March 5-9, 1997). Circulation 2000;102(20):E126-63.

3. Krishnan US, Taneja I, Gewitz M, et al. Peripheral vascular adaptation and orthostatic tolerance in Fontan physiology. Circulation 2009;120(18):1775-83.

4. Zamboni P, Menegatti E, Weinstock-Guttman B, et al. The severity of chronic cerebrospinal venous insufficiency in patients with multiple sclerosis is related to altered cerebrospinal fluid dynamics. Funct Neurol 2009;24(3):133-8.

5. Zamboni P, Menegatti E, Weinstock-Guttman B, et al. CSF dynamics and brain volume in multiple sclerosis are associated with extracranial venous flow anomalies: a pilot study. Int Angiol;29(2):140-8.

6. Zamboni P, Galeotti R, Menegatti E, et al. Chronic cerebrospinal venous insufficiency in patients with multiple sclerosis. J Neurol Neurosurg Psychiatry 2009;80(4):392-9.

7. Al-Omari MH and Rousan LA. Internal jugular vein morphology and hemodynamics in patients with multiple sclerosis. Int Angiol;29(2):115-20.

8. Simka M, Kostecki J, Zaniewski M, et al. Extracranial Doppler sonographic criteria of chronic cerebrospinal venous insufficiency in the patients with multiple sclerosis. Int Angiol;29(2):109-14.

9. Dolic K, Marr K, Valnarov V, et al. Sensitivity and specificity for screening of chronic cerebrospinal venous insufficiency using a multimodal non-invasive imaging approach in patients with multiple sclerosis. Funct Neurol;26(4):205-14. 
10. Simka $M$ and Zaniewski $M$. Reinterpreting the magnetic resonance signs of hemodynamic impairment in the brains of multiple sclerosis patients from the perspective of a recent discovery of outflow block in the extracranial veins. J Neurosci Res;88(9):1841-5.

11. Lagana MM, Forzoni L, Viotti S, et al. Assessment of the cerebral venous system from the transcondylar ultrasound window using virtual navigator technology and MRI. Conf Proc IEEE Eng Med Biol Soc;2011:579-82.

12. Zivadinov R, Galeotti R, Hojnacki D, et al. Value of MR Venography for Detection of Internal Jugular Vein Anomalies in Multiple Sclerosis: A Pilot Longitudinal Study. AJNR Am J Neuroradiol;32(5):938-46.

13. Laupacis A, Lillie E, Dueck A, et al. Association between chronic cerebrospinal venous insufficiency and multiple sclerosis: a meta-analysis. CMAJ;183(16):E1203-12.

14. Zamboni P, Morovic S, Menegatti E, et al. Screening for chronic cerebrospinal venous insufficiency (CCSVI) using ultrasound--recommendations for a protocol. Int Angiol;30(6):571-97.

15. Menegatti E, Genova V, Tessari M, et al. The reproducibility of colour Doppler in chronic cerebrospinal venous insufficiency associated with multiple sclerosis. Int Angiol;29(2):121-6.

16. Doepp F, Paul F, Valdueza JM, et al. No cerebrocervical venous congestion in patients with multiple sclerosis. Ann Neurol 2010;68(2):173-83.

17. Doepp F, Wurfel JT, Pfueller CF, et al. Venous drainage in multiple sclerosis: a combined MRI and ultrasound study. Neurology;77(19):1745-51.

18. Bridges TJ, Clark K and Yahr MD. Plethsymographic studies of the cerebral circulation: evidence for cranial nerve vasomotor activity. J Clin Invest 1958;37(5):763-72.

19. Folkow B and Neil E. Circulation. New York: Oxford University Press, 1973.

20. Ambarki $\mathrm{K}$, Baledent $\mathrm{O}$, Kongolo $\mathrm{G}$, et al. A new lumped-parameter model of cerebrospinal hydrodynamics during the cardiac cycle in healthy volunteers. IEEE Trans Biomed Eng 2007;54(3):483-91.

21. Raksin PB, Alperin N, Sivaramakrishnan A, et al. Noninvasive intracranial compliance and pressure based on dynamic magnetic resonance imaging of blood flow and cerebrospinal fluid flow: review of principles, implementation, and other noninvasive approaches. Neurosurg Focus 2003;14(4):e4. 22. Monti L, Menci E, Ulivelli M, et al. Quantitative ColourDopplerSonography evaluation of cerebral venous outflow: a comparative study between patients with multiple sclerosis and controls. PLoS One;6(9):e25012.

23. Zivadinov R, Poloni GU, Marr K, et al. Decreased brain venous vasculature visibility on susceptibilityweighted imaging venography in patients with multiple sclerosis is related to chronic cerebrospinal venous insufficiency. BMC Neurol;11:128.

24. Mancini M, Morra VB, Di Donato O, et al. Multiple sclerosis: cerebral circulation time. Radiology;262(3):947-55.

25. Zamboni P, Consorti G, Galeotti R, et al. Venous Collateral Circulation Of The Extracranial Cerebrospinal Outflow Routes. Curr Neurovasc Res 2009.

26. Law M, Saindane AM, Ge Y, et al. Microvascular abnormality in relapsing-remitting multiple sclerosis: perfusion MR imaging findings in normal-appearing white matter. Radiology 2004;231(3):645-52.

27. Ge Y, Law M, Johnson G, et al. Dynamic susceptibility contrast perfusion MR imaging of multiple sclerosis lesions: characterizing hemodynamic impairment and inflammatory activity. AJNR Am J Neuroradiol 2005;26(6):1539-47.

28. Varga AW, Johnson G, Babb JS, et al. White matter hemodynamic abnormalities precede subcortical gray matter changes in multiple sclerosis. J Neurol Sci 2009;282(1-2):28-33.

29. Parvey HR, Eisenberg RL, Giyanani V, et al. Duplex sonography of the portal venous system: pitfalls and limitations. AJR Am J Roentgenol 1989;152(4):765-70. 


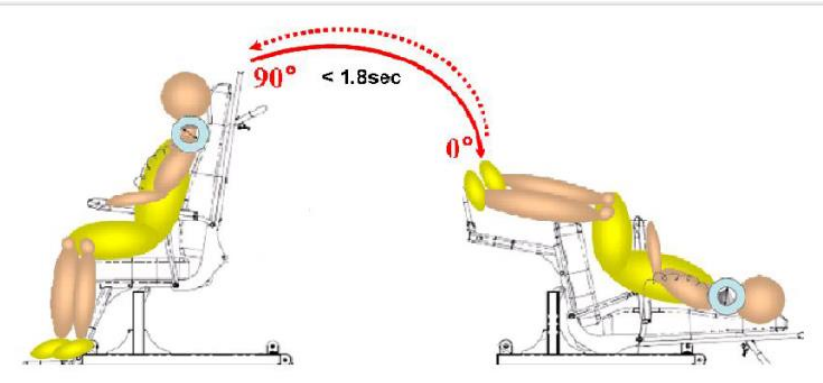

Figure 1. The operator places the strain gauge around the neck of the subject, and rapidly tilts the chair backwards from $90^{\circ}$ to $0^{\circ}$. When the blood volume curve reaches a plateau, the subject is again tilted forward to the upright position $(<1.8 \mathrm{sec})$.

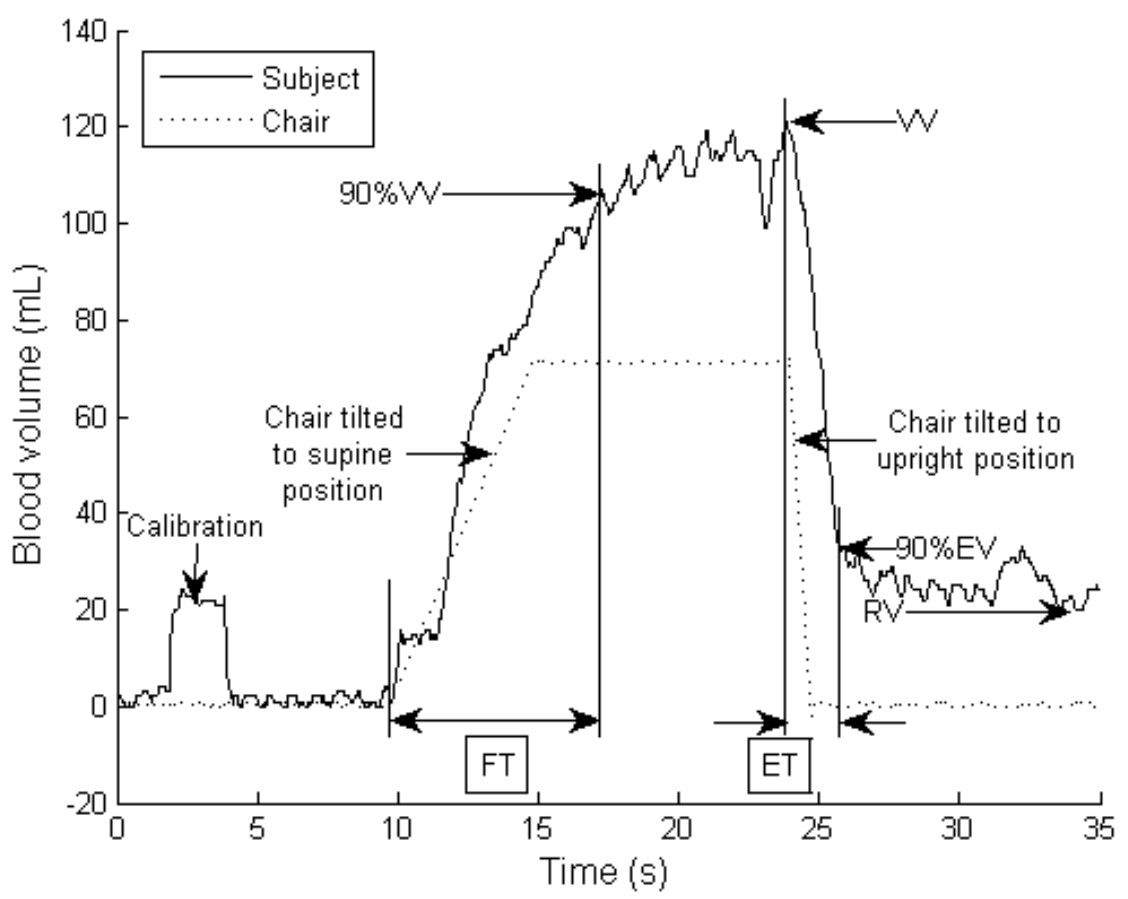

Figure 2. The plethysmographic trace is represented by the solid line, with the gravitational gradient (measured by the sensor positioned on the tilt chair) represented by the dotted line. For each experimental run the following key parameters were recorded: the venous volume (VV); the filling time (FT) required to achieve $90 \%$ of VV $(90 \% \mathrm{VV})$; the residual volume (RV); and the emptying time (ET) required to achieve $90 \%$ of $\mathrm{EV}(90 \% \mathrm{EV})$, where $\mathrm{EV}=\mathrm{VV}-\mathrm{RT}$. 


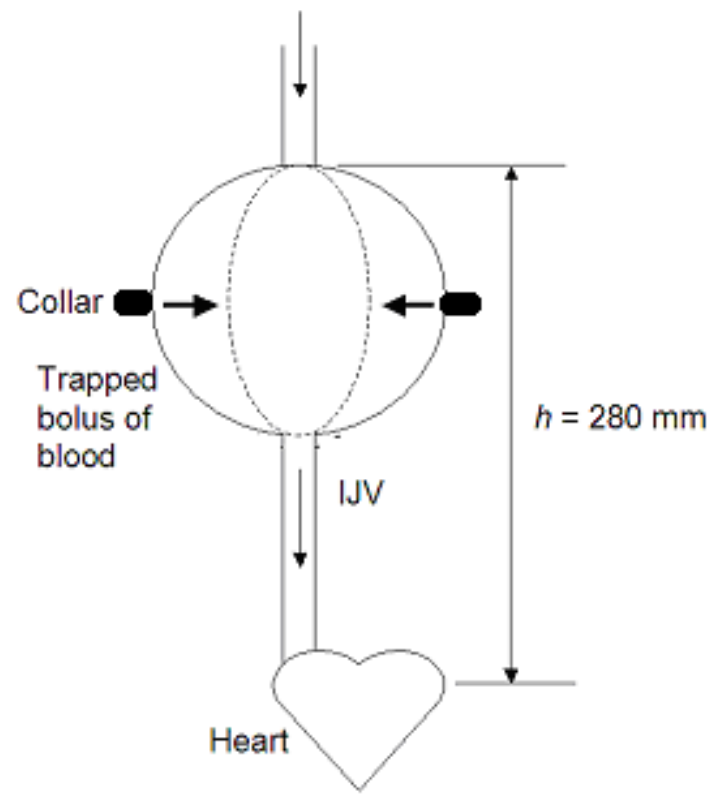

Figure 3. Simplified diagram of plethysmography discharge process in the upright position.
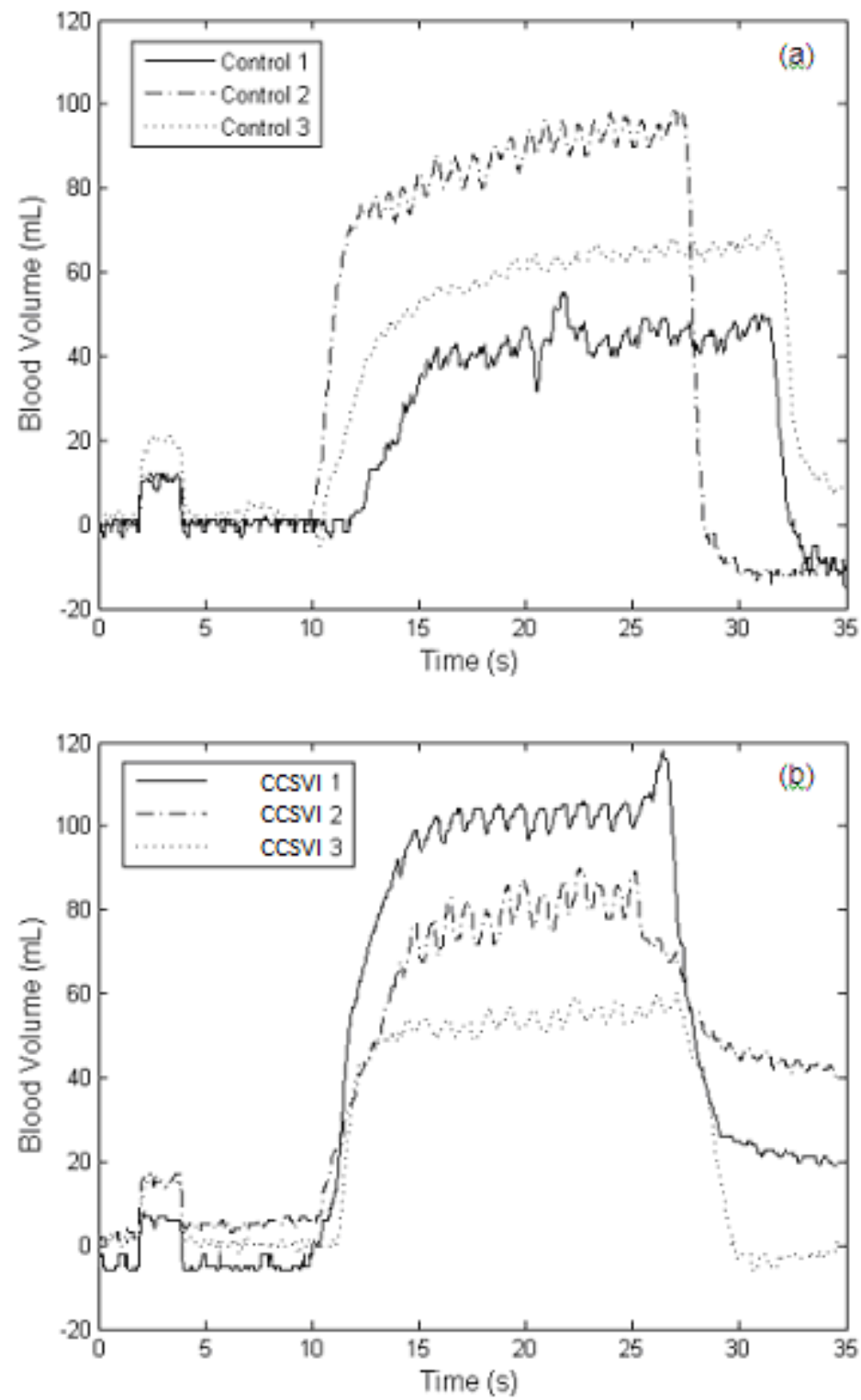

Figure 4. Typical plethysmogram plots for healthy individuals (a) and CCSVI patients (b). 

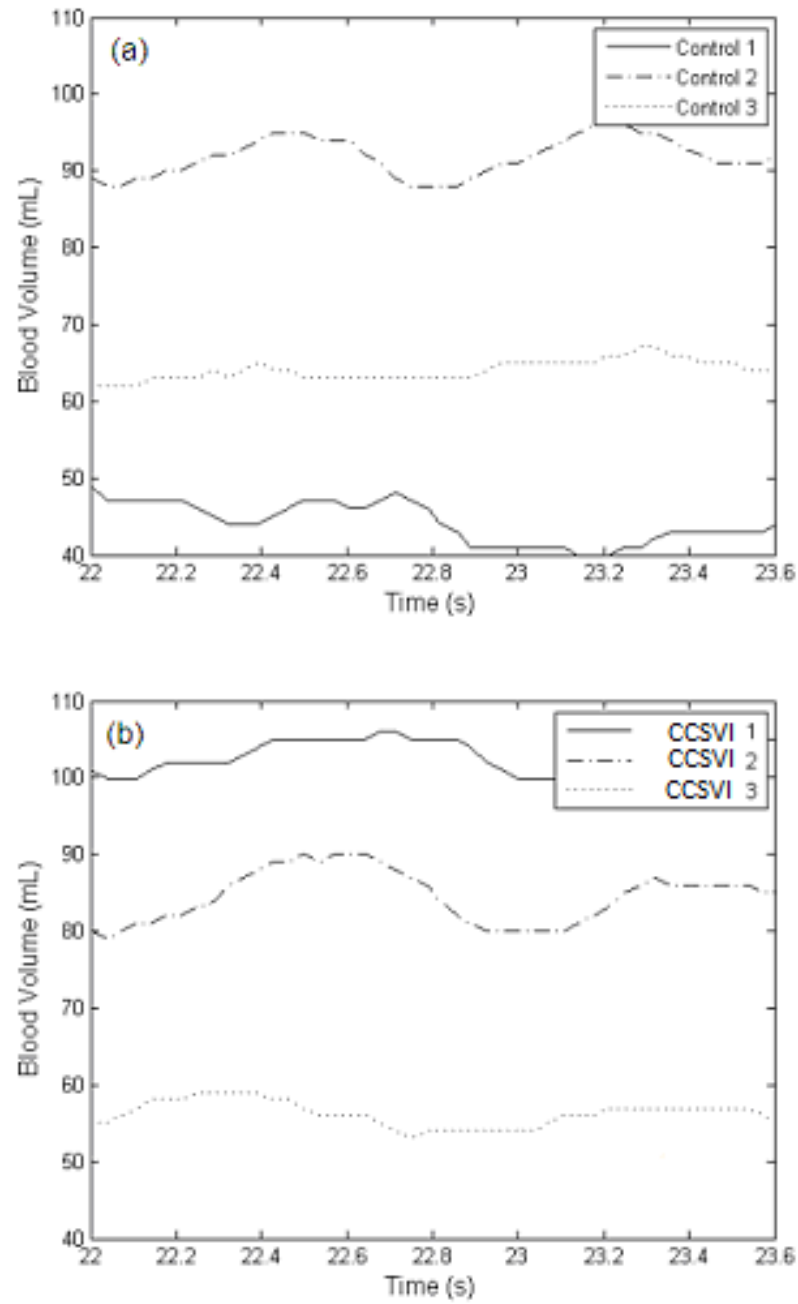

Figure 5. Sample plethysmogram plots for part of oscillatory period: (a) healthy individuals; and (b) CCSVI patients. 


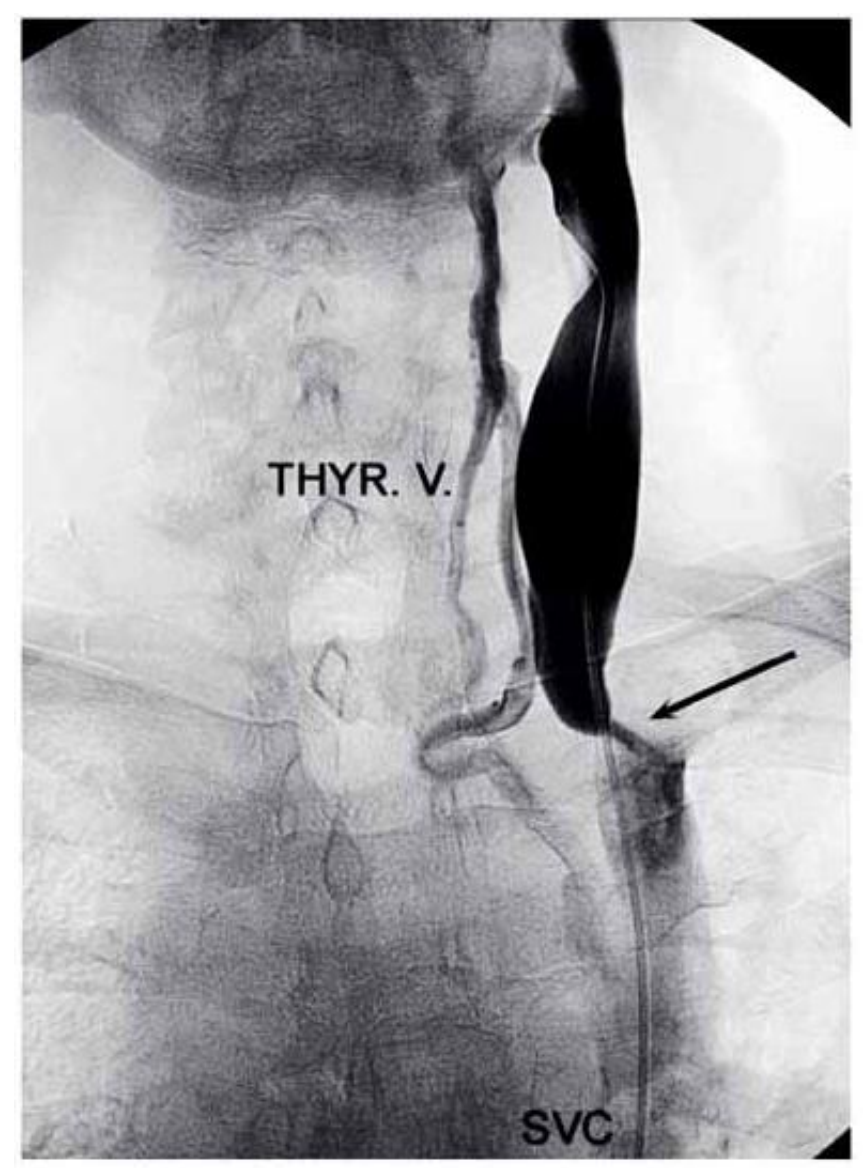

Figure 6. Collateral flow through the thyroid veins in a MS patient due to $95 \%$ stenosis of the left IJV. 